\title{
Gender und Innovationen - Erfahrungen aus dem Projekt „Discover Gender!“
}

NinA BESSING und Helga LuKOschat

Der Artikel skizziert die Erfahrungen des Projekts „Discover Gender“, das die Fraunhofer Gesellschaft von 2003 bis 2006 in einem interdisziplinärem Team durchgeführt hat. Der Artikel geht dabei insbesondere auf die Bedeutung der Gender- und Innovationsforschung für die anwendungsorientierte Forschung und Technikentwicklung ein.

\section{Einleitung}

Inwieweit führt die Integration der Gender-Dimension zu einer erhöhten Innovationsfähigkeit in Forschung und Entwicklung? Welche Blickerweiterung und welche Chancen und Potenziale sind damit für die erhöhte Kundinnen- und Kundenzufriedenheit verbunden? Und: Wie kann den Prozessen der Benachteiligung und Stereotypisierung von Frauen, zum Beispiel in der Produktentwicklung, entgegen gewirkt werden?

Mit diesen Fragen startete das Projekt „Discover Gender“, das von der Fraunhofer Gesellschaft mit Förderung des Bundesministeriums für Bildung und Forschung von 2003 bis 2006 in einem interdisziplinären Projektteam $^{1}$ durchgeführt wurde. Übergreifendes Ziel des Projekts war

1 Die Projektleitung lag bei der Zentrale der Fraunhofer Gesellschaft. Projektpartner waren folgende Organisationen: Europäische Akademie für Frauen in Politik und Wirtschaft (EAF), Fraunhofer Institut System- und Innovationsforschung (ISI), Trommsdorff \& Drüner, Innovation und Marketing Consultans GmbH und Wehking PR. Das Projekt wurde mit Mitteln des Bundesministeriums für Bildung und Forschung gefördert. 
es, Wissensgrundlagen und Methodiken für die Integration von GenderAspekten in die anwendungsorientierte Forschung und Technologieentwicklung zu erarbeiten. Dabei kombinierte das Projekt im Wesentlichen drei Fragestellungen: Erstens ging es darum in ausgewählten Forschungsgebieten der Fraunhofer Gesellschaft Gender-Aspekte zu ermitteln, über konkrete Beispiele zu veranschaulichen und damit die Relevanz von Gender sichtbar zu machen.

Zweitens interessierte uns die Fragestellung wie, mit welchen Prozessen, Instrumenten und Methodiken, es möglich wird, Gender-Aspekte aufzudecken und in Forschungs- und Entwicklungs- (F\&E) Prozesse zu integrieren.

Drittens war es unser Ziel, Forscher und Forscherinnen in der Fraunhofer Gesellschaft zu sensibilisieren und Erfahrungen über geeignete Sensibilisierungsstrategien zu sammeln.

Diese drei Fragestellungen wurden verbunden mit einer innovationspolitischen Perspektive: Das deutsche Innovationssystem steht vor großen Herausforderungen, aber auch vor großen Chancen. Nach wie vor gehört Deutschland zu den im internationalen Vergleich innovativsten Volkswirtschaften. Neuere Studien weisen allerdings darauf hin, dass das deutsche Innovationssystem seine Leistungsfähigkeit erhöhen könnte, wenn es gelingen würde,

- die inhaltlichen Potenziale der Gender-Forschung für die Qualität und Bedarfsgerechtigkeit anwendungsorientierter Forschung und Technikentwicklung fruchtbar $\mathrm{zu}$ machen (vgl. Schraudner/Lukoschat 2006; Buhr 2006) und

- die Potenziale und Begabungen von Hochschulabsolventinnen und Forscherinnen für das Innovationsmanagement und für Forschungsund Entwicklungsprozesse in der Industrie besser als bisher zu erschließen (vgl. Deutsche Telekom Stiftung 2005; Europäische Kommission 2006).

Die innovationspolitische Bedeutung von Gender-Aspekten in der anwendungsorientierten Forschung und Technikentwicklung enthält also eine inhaltliche und eine personalpolitische Dimension.

Bei der inhaltlichen Dimension, wie wir sie im Projekt verstanden haben, geht es um die Integration der Erfahrungen und Theorien der Gender-Forschung in anwendungsorientierte Forschung- und Technikentwicklung und damit letztendlich um eine gendersensible Erweiterung des Blickfeldes. Die personalpolitische Dimension bezieht sich vor allem auf die Förderung und die Integration von Frauen in anwendungs- 
orientierte Forschung und Technikentwicklung und die damit verbundenen Maßnahmen zur Personal- und Organisationsentwicklung.

Die meisten Gender-Projekte bezogen sich in der Vergangenheit auf die personalpolitische Dimension, daher startet das Projekt „Discover Gender!“ ganz klar mit dem Ziel, die inhaltliche Dimension zu beleuchten.

Im vorliegenden Beitrag werden die Erfahrungen, die wir im Laufe des Projekts gemacht haben, dargelegt.

\section{Zum Grundverständnis von Gender}

Die Auseinandersetzung mit grundsätzlichen Fragen der GenderForschung begleitete sowohl uns als interdisziplinäres Projektteam als auch unsere Arbeit mit den Forscherinnen und Forschern der Fraunhofer Gesellschaft von Anfang an. Unser definiertes Ziel war es, durch den Einbezug der Gender-Dimension einen Beitrag zur Qualitätserhöhung anwendungsorientierter Forschung und Technikentwicklung zu leisten. Ziel war es auch, die gleiche Teilhabe von Frauen und Männern an der Entwicklung technischer Lösungen $\mathrm{zu}$ ermöglichen und damit letztendlich vielfältige und bedarfsgerechte Nutzungsoptionen von Technologien auch jenseits traditioneller Geschlechtsrollen zu ermöglichen.

Die theoretische wie praktische Herausforderung ist, GenderAspekte in anwendungsorientierter Forschung und Technikentwicklung zu berücksichtigen, ohne Stereotype und Rollenmuster zu reproduzieren. Unsere Erfahrung in dem Projekt hat gezeigt, dass dies vor allem bedeutet, den Begriff Gender und seine Bedeutung im jeweiligen Forschungskontext beständig zu reflektieren und Gender einzubetten in ein umfassenderes Konzept von Diversität. Je nach Forschungsfeld und Themenstellung können mehrere Differenzierungsmerkmale wie Nationalität, Alter oder berufliche Sozialisation relevant sein. So zeigte sich z.B. bei einem unserer „Nutzer/innen-Workshops“, der die Testung eines neu entwickelten Prototyps zur Bluthochdruckmessung zum Ziel hatte, dass die Anforderungen an das Gerät maßgeblich von Alter, Geschlecht und Beruf der Nutzerinnen und Nutzer bestimmt wurde. Geschlechtsunterschiede allein waren nicht in der Lage die unterschiedlichen Präferenzen zu erklären, doch ohne den Einbezug der Variable Geschlecht hätten wir z.B. nicht herausfinden können, dass die befragten Nutzerinnen bereit waren mehr Geld für das Bluthochdruckgerät auszugeben als die befragten Nutzer. Dabei gilt es, den Blick nicht nur auf individuelle Geschlechtsunterschiede, sondern auch auf die organisationale und gesellschaftliche Ebene zu lenken und den Zusammenhang zwischen diesen 
verschiedenen Ebenen zu berücksichtigen. In einem Workshop mit Forscherinnen und Forschern aus der Verkehrs- und Umweltforschung betrachteten wir beispielsweise die Ergebnisse der Forschung zu gesellschaftlichen Mobilitätsmustern und leiteten daraus Schlussfolgerungen für die konkreten Projekte der Forscherinnen und Forscher ab: Frauen sind immer noch stärker für Hausarbeit und Familienarbeit zuständig und legen daher im Durchschnitt pro Tag viele eher kürzere Wegstrecken zurück und gehen häufiger zu Fuß oder benutzen das Fahrrad. Männer sind häufig Vollzeit erwerbstätig und legen daher eher wenige und längere Wegstrecken zurück. Diese unterschiedlichen Mobilitätsmuster resultieren nicht aus einem generellen Unterschied zwischen Frauen und Männern, sondern aus gesellschaftlichen Anforderungen an Erwerbstätigkeit und Reproduktionsarbeit (Klima-Bündnis/Stete Planung 2001, zit.n. Genanet 2001:1). Beide Formen der Arbeit sind für die Gesellschaft notwendig und sollten daher bei der Entwicklung von nutzungsgerechten Verkehrssystemen berücksichtigt werden.

Der Einbezug des sozialen Kontextes lässt auch die Kopplung von Geschlechtszugehörigkeit mit Diskriminierungen auf gesellschaftlicher und/ oder organisationaler Ebene sichtbar werden. Diese Zusammenhänge den Forscherinnen und Forschern aufzuzeigen war ebenfalls wichtig. Die konstruktivistische Perspektive in der Geschlechterforschung macht deutlich, dass Geschlechtsunterschiede zum großen Teil auch ,gemachte Geschlechtsunterscheidungen“" sind (Krell 2004: 27), die als Legitimierung für Diskriminierungen dienen können. Wenn wir Gender-Aspekte in FuE-Projekten betrachten, reicht es also nicht aus, die Unterschiede zwischen Männern und Frauen zu berücksichtigen. Im Gegenteil: Gender-Aspekte können sich auch dort zeigen, wo bisher von Unterschieden, z.B. im Sinne von Rollenklischees, ausgegangen wird, obwohl vielmehr Gemeinsamkeiten zwischen den Geschlechtern bestehen. Es gilt also immer Unterschiede und Gemeinsamkeiten in den Blick zu nehmen. Dies zeigte sich z.B. bei der Befragung von Probandinnen und Probanden zur Testung eines tragbaren PDA (Personal Digital Assistant) auf der Bundesgartenschau 2006 (BuGa) in München. Der so genannte Buga-Butler stellte Informationen für die Besucherinnen und Besucher der BuGa bereit. Zu Beginn der Produktentwicklung waren die Forscher und Forscherinnen davon ausgegangen, dass die weibliche Hauptnutzerinnengruppe Frauen zwischen 50 und 65 Jahren mit wenig technischen Erfahrungen sind. Im Verlauf der Untersuchung stellte sich heraus, dass die weiblichen Nutzerinnen des Buga-Butlers eher der Gruppe der technikaffinen Frauen zuzurechnen waren. Die Gemeinsamkeiten zwischen weiblichen und männlichen Nutzern bezüglich der Technikaffinität waren größer als erwartet. 


\section{Gender und Innovation}

Innovations- und Gender-Forschung kommen unabhängig voneinander zu zum Teil vergleichbaren Einschätzungen bezüglich des Veränderungsbedarfs in der industriellen Forschung- und Technikentwicklung. Doch stehen die beiden Stränge bisher unverbunden nebeneinander. Gender-Forschung findet zumeist ohne Einbezug von Ergebnissen der Innovationsforschung statt; die Innovationsforschung wiederum integriert nicht die Gender- und Diversity-Dimension.

Zumeist wird der Begriff des Innovationsprozesses im Innovationsmanagement im weiten Sinne gebraucht und umfasst sowohl die gesamte F\&E als auch das Anfahren der Produktion und die erfolgreiche Markteinführung eines neuen Produktes oder einer neuen Dienstleistung. Aus dieser Perspektive ist die anwendungsorientierte Forschung und Technikentwicklung in der Fraunhofer Gesellschaft, die Gegenstand unseres Projekts war, die erste Phase eines Innovationsprozesses. Dabei kann es sich bei der Innovation um eine objektive Neuheit bzw. Weltneuheit handeln, wenn die Ergebnisse erstmals in dieser Form entwickelt wurden. Eine subjektive Neuheit bzw. Betriebsneuheit liegt dagegen vor, wenn das Ergebnis nur für die Entscheidungsträger und Entscheidungsträgerinnen neu ist (Specht et al. 2002: 14).

\section{„KundInnen“- und Marktorientierung}

Bei der Frage nach der geeigneten strategischen Ausrichtung des Innovationsmanagements wird oft diskutiert, ob grundsätzlich eine ,Technology Push“-Strategie oder eine ,Demand Pull“-Strategie wichtiger ist. Dabei wird unter „Technology Push“ verstanden, ein latent vorhandenes Bedürfnis potenzieller Kunden und Kundinnen durch die Entwicklung einer Innovation zu wecken. Die „Demand Pull“ Strategie geht dagegen davon aus, dass Entwicklungsaktivitäten durch die Nachfrage am Markt induziert werden und daher vornehmlich an dessen Anforderungen zu orientieren sind. Die bisherigen Studien zu den Erfolgsfaktoren von Innovationsprojekten zeigen, dass insbesondere bei Projekten mit inkrementalem Neuheitsgrad die marktorientierte Strategie sich als erfolgreicher erwiesen hat als die technologieorientierte Strategie. Dagegen spielt insbesondere bei radikalen technischen Neuerung die technologieorientierte Strategie ebenfalls eine wichtige Rolle (Specht et. al 2002: 32). Grundsätzlich lässt sich aber auf Basis des jetzigen Forschungsstandes das Fazit ziehen: Es besteht ein positiver Zusammenhang zwischen Markterfolg und Kundenorientierung, auch beim Management der frü- 
hen Innovationsphasen, d.h. auch für Forschungs- und Entwicklungsprozesse (Lüthje 2003: 37).

Genau diese Kundenorientierung ist ein wichtiger Anknüpfungspunkt für die Integration von Gender-Aspekten in anwendungsorientierte Forschungs- und Technikentwicklung: Die Kundenorientierung schärft das Bewusstsein dafür, bereits in der Technologie- und Produktentwicklung den Blick frühzeitig auf unterschiedliche Kundengruppen zu richten, ihre Zugangsweisen und Präferenzen zu analysieren und den jeweiligen sozialen oder kulturellen Nutzungskontext einzubeziehen. Unter den Bedingungen der Globalisierung verändern sich die Märkte zum Teil rapide, aber auch andere soziale und ökonomische Entwicklungen beeinflussen und beschleunigen den Wandel. Dazu gehört nicht zuletzt der Wandel des Geschlechterverhältnisses, der sich zur Zeit in allen großen Industrienationen vollzieht und in Deutschland u.a. an der wachsenden Bildungs- und Erwerbsbeteiligung von Frauen abzulesen ist, und an der seit Jahren intensiv geführten Debatte um die neue, partnerschaftliche Rollen- und Aufgabenverteilungen zwischen Frauen und Männern.

Dabei lassen sich in Bezug auf Gender drei markrelevante Trends feststellen, die in anwendungsorientierter Forschung und Technikentwicklung relevant sein können (vgl. Brühl/Westphal 2004; Horx 2003; Jaffé 2005):

- Der Bedeutungszuwachs von Kundinnen und Konsumentinnen: Die Marktmacht von Frauen steigt und damit steigt die Notwendigkeit, sich mit ihren Präferenzen auseinanderzusetzen. Studien zeigen, dass Frauen häufig sehr viel höhere Ansprüche an das Design, die Nutzungsfreundlichkeit von Technik und den Service technischer Dienstleistungen haben (vgl. Horx 2003).

- Das Aufbrechen traditionell weiblicher und männlicher Märkte: Die traditionelle Aufteilung - Frauen entscheiden über die Konsumgüter des täglichen Bedarfs und Männer über die größeren Anschaffungen - gilt heute nicht mehr. Frauen erobern die Märkte für Technikprodukte und Männer werden in ehemals traditionellen Frauenmärkten, wie z.B. der Kosmetikindustrie, zu einer relevanten Größe. In Paarbeziehungen und Familien wird heute ein immer größerer Teil der Kaufentscheidungen gemeinsam getroffen. D.h. auch hier verändern sich die Entscheidungsmuster, nach denen technische Lösungen am Markt nachgefragt werden. ${ }^{2}$

2 U.a. hat das in Deutschland, Österreich und der Schweiz ansässige Marktforschungsinstitut SevenOne Media eine Studie zu den veränderten Lebens- und Konsumgewohnheiten von Frauen und Männern unter dem Titel „Trendreport Frauen“ herausgebracht und dabei vor allem die Bereiche 
- Die Individualisierung und Ausdifferenzierung von Kundenbedürfnissen: Der Trend geht weg von reinen „Frauen“- oder „Männer“Produkten zur Individualisierbarkeit und Anpassungsfähigkeit von Produkten. Es gilt, sich der vielfältigen Lebensentwürfe und -formen von Frauen und Männern bewusst zu werden und Klischees und Stereotype zu vermeiden. Nur ein Beispiel dafür ist der Bereich der Heimwerker- und Baumärkte, in denen Frauen mittlerweile knapp die Hälfte der Kunden stellen und neuartig gestaltete Werkzeuge mit geringerem Gewicht und für kleine Hände (z.B. Bohrmaschinen oder Akkuschraubenzieher) große Markterfolge erzielen und zwar auch bei Männern. ${ }^{3}$

Im Projekt „Gender-Aspekte in der Forschung“ lag deshalb auch ein Schwerpunkt im Bereich der Marktanalyse mit der Frage, welche Methoden und Instrumente notwendig sind, um die neuen Trends zu einer komplexeren und differenzierteren Lebenssituation von Frauen und Männern tatsächlich erfassen und in Forschungs- und Technikentwicklung integrieren zu können (für eine Vertiefung sei hier auf den Sammelband „Gender als Innovationspotenzial in Forschung und Entwicklung“" von Martina Schraudner und Helga Lukoschat verwiesen).

\section{Interdisziplinarität und Vielfalt}

Es existiert ein weiterer wichtiger Anknüpfungspunkt in der aktuellen Diskussion um die Innovationsfähigkeit von Organisationen, der die Integration von Gender-Aspekten sinnvoll erscheinen lässt. Es besteht unter Innovationsmanagement-Expertinnen und -Experten Einigkeit darüber, dass Perspektivenvielfalt und Interdisziplinarität in Forschungsund Entwicklungsteams eine der wichtigsten Grundvoraussetzungen ist um Innovationen hervorzubringen. In zahlreiche Veröffentlichungen in den letzten Jahren wird die vielfältige Zusammensetzung von F\&E- und Innovationsteams als Erfolgsgeheimnis bezeichnet und es wurden zahlreiche Methodiken zur Integration von Perspektivenvielfalt entwickelt (vgl. z.B. Kelley 2001; Kelley 2005; Schnetzler 2006; Peters 2003). Die Gender-Forschung kann dazu einen wichtigen Beitrag leisten: Zahlreiche Studien weisen nach, dass bei homogen zusammengesetzten For-

Automarkt, Telekommunikation, E-Commerce und Finanzen in den Blick genommen. (SevenOne Media 2006).

3 In der erwähnten Studie „Trendreport Frauen“ wird eine von dem Baumarkt Hornbach in Auftrag gegebene Forsa-Untersuchung erwähnt, in der deutlich wird, dass Frauen sich mittlerweile eine Vielzahl von Heimwerkeraktivitäten zutrauen (SevenOne Media 2006: 51). 
scherteams, die keinerlei Methodiken zur Perspektiverweiterung einsetzen, die Gefahr besteht, dass Ergebnisse produziert werden, die nur ungenügend den Anforderungen des Marktes und des realen Anwendungskontextes entsprechen. Daran anknüpfend sind in der Gender-Forschung Grundsätze und Methodiken entwickelt worden, um mehr Perspektivenvielfalt in den Forschungsprozess zu integrieren (vgl. z.B. Buhr/Helmers 1994; Wajcman 1994; Schiebinger 2000; Maaß et al. 2002).

Grundsätze gendersensibler Methoden Zusammenfassend kann aus der Gender-Perspektive für die Technikentwicklung festgehalten werden, dass sich - neben definierten Zielen - Annahmen über Benutzer und Benutzerinnen, Benutzungsweisen und Nutzungskontexten in den technischen Lösungen niederschlagen. Diese Annahmen sind nicht selten - implizit oder explizit - von geschlechtsstereotypen und diskriminierenden oder benachteiligenden Vorstellungen über die Geschlechter geprägt. Diese Vorstellungen fließen in die Designentscheidung ein. Die technischen Lösungen ihrerseits setzen damit Rahmenbedingungen, strukturieren Nutzungsweisen und können auf diese Weise die traditionelle Arbeitsteilung fortschreiben. Technikentwicklung dagegen, die vielfältige Nutzungskontexte explizit berücksichtigt, kann neue Nutzungsoptionen entwickeln und die Qualität und Bedarfsgerechtigkeit von Lösungen erhöhen. Damit können Diskriminierungen abgebaut und zum Teil sogar neue Markt- und Innovationspotenziale erschlossen werden.

Daran anknüpfend wurden im Projekt „Discover Gender“ Methodiken aus der Innovationsforschung und der Gender-Forschung erprobt und weiter entwickelt, die bei den im letzten Kapitel beschriebenen Herausforderungen Abhilfe schaffen können. Dabei destillierten sich folgende Grundsätze einer gendersensiblen und innovationsförderlichen F\&E heraus:

\section{Präferenzen der Zielgruppen analysieren}

Bei anwendungsorientierten Forschungsvorhaben und Produktentwicklungen wird zumeist erst viel zu spät analysiert, welche Personengruppen als Adressaten bzw. künftige Nutzerinnen und Nutzer der Produkte und Dienstleistungen in Frage kommen und wie deren Präferenzen genau aussehen. Hier zeigen Untersuchungen, dass sich die von Forschern und Forscherinnen antizipierten Präferenzen überproportional häufig an den eigenen Bedürfnissen orientieren und oft nur die Perspektive des vollerwerbstätigen, weißen, männlichen Akademikers mittleren Alters widerspiegeln. Forscher und Forscherinnen und Entwickler und Entwicklerinnen arbeiten häufig immer noch mit unüberprüften Annahmen 
über ihre Kundinnen und Kunden. Je genauer und realitätsbezogener aber diese Charakterisierung erfolgt, desto zielgenauer kann das Forschungs- und Entwicklungsdesign aufgebaut werden. Unter der GenderPerspektive ist zu prüfen, ob Frauen und Männer in der Zielgruppe vertreten sind und welche vielfältigen Bedürfnisse und Präferenzen sich daraus ergeben. Hier stehen insbesondere aus dem Innovationsmanagement zahlreiche Methodiken bereit - von Empathic Design über Focusgruppen bis zu Lead-User-Methodiken - die unter Einbezug von Gender- und Diversity-Aspekten angewandt werden können (vgl. Schraudner/Lukoschat 2006).

\section{Anwendungs- und Nutzungskontext analysieren}

Bei der Analyse des Anwendungs- und Nutzungskontextes eines zu entwickelnden Produktes/Prozesses können Gender-Aspekte wirksam sein. Entsprechend berücksichtigt die Gender-Perspektive, in welchen Lebens- und Arbeitsbereichen und auf welche Weise Männer und Frauen das Produkt bzw. die Technologie bereits tatsächlich anwenden und welche Nutzungsoptionen ein Produkt oder eine Technologie darüber hinaus für Frauen und Männer zukünftig eröffnen könnte. Unsere Befragung von Expertinnen im Rahmen des Projekts ergab, dass hier bisher vornehmlich Nutzungskontexte aus der Erwerbssphäre und der Freizeit in den Blick genommen werden. Die Nutzungskontexte von Familienarbeit, Kinderbetreuung, Pflege älterer Angehöriger etc. werden bislang vernachlässigt.

Auch die gesellschaftlichen Rahmenbedingungen, die ja Anwendungsund Nutzungskontexte maßgeblich prägen, wie z.B. der Rollenwandel der Geschlechter, die zunehmende Erwerbstätigkeit von Frauen, die demografische Entwicklung etc., werden noch zu selten berücksichtigt.

In diesem Zusammenhang gilt auch, dass das Wissen aus sozial-, wirtschafts- und geisteswissenschaftlichen Disziplinen sehr fruchtbar für die Natur- und Ingenieurwissenschaften sein kann. Inter- und Transdisziplinarität erhält daher eine wachsende Bedeutung.

\section{Partizipative Methodiken}

Ein weiteres Potenzial zur Steigerung der Qualität und der InnovationsFähigkeit von Forschung und Entwicklung stellt der frühzeitige Einbezug der Nutzerinnen und Nutzer in den Forschungs- und Entwicklungsprozess dar. Hier gibt es mittlerweile eine Vielzahl an Methodiken, die aber in den seltensten Fällen angewandt werden, um dezidiert die Bedürfnisse von Frauen und Männern zu erfassen. Partizipative und gendersensible Entwicklungsmethoden stellen ein wichtiges Instrumentari- 
um dar, um das Know-how zur Verbesserung von Prozessen und Produkten von Nutzerinnen und Nutzern besser in den F\& E-Prozess zu integrieren. Gendersensible, partizipative Verfahren gehen aber über die Integration von Nutzern und Nutzerinnen hinaus. Die Integration der Erkenntnisse partizipativer Verfahren in Instrumente des Innovationsmanagements kann hier interessante Impulse liefern, denn partizipative Methodiken beziehen alle von den Ergebnissen betroffenen Beteiligten ein und berücksichtigen Faktoren wie die Kommunikationssituation zwischen den Projektbeteiligten und die jeweilige Organisationsstruktur und -kultur. So können beispielsweise in einem Workshop bei der Neuentwicklung einer Software für eine Organisation nicht nur die unmittelbaren Auftrageber (zumeist das Management) einbezogen werden, sondern alle, die direkt oder indirekt von der neuen Software betroffen sein werden. Dies können die Kundinnen und Kunden der Organisation sein, die Anwenderinnen und Anwender der Software aber auch andere Beschäftigte, die über Erfahrungswissen verfügen, das für die Entwicklung der Software relevant ist. Hierdurch kann die Passgenauigkeit und Bedarfsgerechtigkeit von Produkten und Prozessen verbessert werden.

Dennoch sollte die Perspektive der Nutzer und Nutzerinnen nicht verabsolutiert werden. Denn auch die Nutzer und Nutzerinnen sind geprägt durch soziale Strukturen in der Organisation und in der Gesellschaft und durch symbolische und kulturelle Faktoren (vgl. Hammel 2003: 59). Ein vergleichbares Argument wird in der Innovationsforschung diskutiert, wenn darauf hingewiesen wird, dass die Erfassung der Bedürfnisse von Nutzern und Nutzerinnen nicht ausreicht, um neue Märkte zu erschließen, weil Nutzer und Nutzerinnen häufig nur das sehen und beschreiben, was sie bereits kennen und gewohnt sind (Herstatt/Verworn 2003: 42f.). Daraus folgt, dass die Grenzen partizipativer Methodiken dort beginnen, wo Geschlechtsstereotype von den Nutzern und Nutzerinnen selbst reproduziert werden. Um einerseits Diskriminierungen abzubauen und andererseits Visionen von neuen Feldern zu entwickeln, bedarf es immer der differenzierten Reflexion und Weiterentwicklung auch der Kategorie Gender durch den Forscher und die Forscherin. 


\section{Fazit}

Bisher existieren nur wenige Forschungsprojekte, die sich mit der inhaltlichen Gender-Dimension im Bereich anwendungsorientierter Forschung und Entwicklung auseinandergesetzt haben. Notwendig sind mehr Forschungsergebnisse und Good Practice-Beispiele, die den fachlichen und wissenschaftlichen Nutzen der Berücksichtigung von Gender-Aspekten fachspezifisch nachweisen und veranschaulichen. Für die anwendungsorientierte Forschung und Entwicklung konnte das Projekt „Discover Gender" hier erste Ergebnisse generieren.

Die Erfahrungen des Projekts zeigen, dass die Erfassung der Genderund Diversity-Perspektive sehr gut über folgende Maßnahmen erfolgen kann:

- den Einbezug aktueller Ergebnisse der Gender- und Diversity Forschung,

- den direkten Einbezug von Betroffenen durch partizipative Methodiken (z.B. in Innovationswerkstätten oder Nutzer- und Nutzerinnenworkshops) und

- durch den Einsatz von Methodiken aus dem Innovationsmanagement und dem Innovationsmarketing (z.B. „empathic design“ oder LeadUser-Methodiken), die insbesondere unter Einbezug von Genderund Diversity-Aspekten angewandt werden.

Ziel eines gender- und diversitysensiblen Innovationsmanagements ist nicht allein, die Perspektive von Frauen stärker in den Prozess zu integrieren, sondern auch die Vielfalt an Bedürfnissen und Erwartungen an neu entwickelte Produkte, Prozesse und Dienstleistungen seitens der Kunden und Kundinnen in den Blick zu nehmen und zu berücksichtigen. So können Produkte und Dienstleistungen an neue Bedürfnisse angepasst und Fehlentwicklungen vermieden werden. Innovationen erhalten eine höhere Marktakzeptanz und es lassen sich neue Marktpotenziale erschließen. Die Individualisierbarkeit und Bedarfsgerechtigkeit der Lösungen wird verbessert und Forscherinnen und Forscher erschließen neue, vielfältige Nutzungsoptionen. 


\section{Literatur}

Buhr, Regina (Hg.) (2006): Innovationen - Technikwelten, Frauenwelten, Berlin.

Buhr, Regina/Helmers, Sabine (1994): Corporate Story-Telling. The Buxomly Secretary, a Pyrrhic Victory of the Male Mind. In: Scandinavian Journal of Management, Vol. 10, Nr. 2, S. 175-191.

Brühl, Kirsten/Westphal, Susanne (2004): Female Forces - Der Megatrend Frauen und seine Auswirkungen auf Konsum und Business. Studie der Zukunftsinstitut GmbH Kerkheim, München.

Europäische Kommission (Hg.) (2006): Women in science and technology - the business perspective, Brüssel.

Deutsche Telekom-Stiftung (Hg.) (2005): Innovationsindikator Deutschland 2005 - Ergebnisse einer Studie des DIW, Köln/Berlin.

Genanet (2001): Kurzinformation zu Gender und Nachhaltigkeit, Nr. 5: Mobilität, www.genanet.de.

Hammel, Martina (2003): Partizipative Softwareentwicklung im Kontext der Geschlechterhierarchie, Frankfurt am Main.

Herstatt, Cornelius/Verworn, Birgit (Hg.) (2003): Management der frühen Innovationsphasen. Grundlagen - Methoden - Neue Ansätze, Wiesbaden.

Horx, Matthias (2003): Accent on the Future, Zukunftsstudie von Accenture, Wien.

Jaffé, Diana (2005): Der Kunde ist weiblich, Berlin.

Kelley, Tom (2001): The Art of Innovation, Currency Doubleday, New York.

Kelley, Tom (2005): The Ten Faces of Innovation, Currency Doubleday, New York.

Klima-Bündnis/Stete Planung (2001): Frauen bewegen die Stadt. Daten - Fakten - Argumente, Frankfurt am Main.

Krell, Gertraude (2004): Chancengleichheit durch Personalpolitik, 4. Auflage, Wiesbaden, S. 27ff.

Lüthje, Christian (2003): Methoden zur Sicherstellung von Kundenorientierung in den frühen Phasen des Innovationsprozesses. In: Management der frühen Innovationsphase, hg.v. Cornelius Herstatt/Birgit Verworn, Wiesbaden, S. 35-56.

Maaß, Susanne/Theißing, Florian/Zallmann, Margita (2002): Unterstützung von Interaktionsarbeit im Call-Center. Neue Fragen für die arbeitsorientierte Softwareentwicklung. In: $i$-com. Zeitschrift für interaktive und kooperative Medien, 3/2002, S. 4-11.

Peters, Tom (2003): Re-imagine! Business Excellence in a Disruptive Age, Dorling Kindersley Limited, London. 
Schraudner, Martina/Lukoschat, Helga (Hg.) (2006): Gender als Innovationspotenzial in Forschung und Entwicklung, Stuttgart.

Schiebinger, Londa (2000): Frauen forschen anders. Wie weiblich ist die Wissenschaft? München.

Schnetzler, Nadja (2006): Die Ideenmaschine: Methode statt Geistesblitz - Wie Ideen industriell produziert werden, Weinheim.

SevenOne Media (2006): TrendReport Frauen, München-Unterföhring.

Specht, Günter/Beckmann, Christoph/Amelingmeyer, Jenny (2002):

F\&E-Management - Kompetenz im Innovationsmanagement, 2. Auflage, Stuttgart.

Wajcman, Judy (1994): Technik und Geschlecht. Die feministische Technikdebatte, Frankfurt am Main. 
